\title{
Mixed glaucoma
}

\author{
S. W. HYAMS, C. KEROUB, AND E. POKOTILO \\ From the Department of Ophthalmology, Central Emek Hospital, Afula, Israel
}

SUMmaRY Two hundred and sixty-seven eyes which underwent peripheral iridectomy for presumed angle-closure glaucoma were investigated. The diagnosis of angle-closure glaucoma was confirmed postoperatively in 258 eyes, in 201 of which the intraocular pressure became normal without further treatment and in 57 of which the postiridectomy IOP was elevated because of visible damage to the anterior chamber angle. Three eyes had open-angle glaucoma with a narrow angle. Six eyes had possible mixed (combined mechanism) glaucoma $(2 \cdot 2 \%)$. Mixed glaucoma is a rare, fortuitous combination of open-angle and angle-closure glaucoma rather than a distinct entity, and it might be advisable to avoid the term, which tends to be used for primary glaucomas of uncertain classification.

The primary glaucomas have been classified into 3 groups: open-angle glaucoma, angle-closure (narrow-angle) glaucoma, and combined mechanism or mixed glaucoma (Shaffer, 1960). Since the heredity and pathogenesis of open-angle glaucoma and of angle-closure glaucoma are completely different, their coexistence should be a rare, chance combination. On the other hand most physicians would agree that the diagnosis of mixed glaucoma is made not uncommonly, especially when the differential diagnosis between oren-angle and angleclosure glaucoma is uncertain.

A promising method for assessing the incidence of mixed glaucoma is to study eyes which have undergone peripheral iridectomy: if angle-closure glaucoma was definitely present before the iridectomy and if glaucoma was still present postiridectomy in spite of an open, normal angle, the diagnosis of mixed glaucoma is probable.

We could find no report of such an investigation in the literature.

\section{Materials and methods}

The records of 159 consecutive patients who underwent peripheral iridectomy for (presumed) primary angle-closure glaucoma were reviewed. Patients in whom the intraocular pressure (IOP) was higher than $20 \mathrm{mmHg}$ on any occasion following iridectomy and in whom the elevated IOP was not clearly due to the presence of goniosynechiae obliterating a significant part of the angle were recalled for study. The examination of these patients consisted of applanation tonometry without and with treatment, examination of the optic disc and visual fields, and detailed gonioscopy. In most cases gonioscopy was performed by two physicians.

Altogether 267 eyes of 159 patients underwent peripheral iridectomy. There were 89 males and 70 females; 95 eyes had had acute angle-closure glaucoma, 125 eyes had (presumed) intermittent or chronic ('creeping') angle-closure glaucoma, and 47 eyes were fellow eyes of patients who had suffered acute glaucoma in one eye.

The results of peripheral iridectomy are summarised in the table. Of the 267 eyes 258 have pure, angle-closure glaucoma. In 201 of these eyes the IOP became normal after iridectomy, in 42 eyes the IOP remained elevated after iridectomy owing to the presence of extensive goniosynechiae, and in 15 eyes the IOP remained elevated and gonioscopy showed visible damage to the angle in the absence of goniosynechiae. This damage consisted of irregularly distributed, coarse granules and patches

\section{Table Results of peripheral iridectomy in 267 eyes}

\begin{tabular}{lll}
\hline & Eyes & $\begin{array}{c}\text { Eyes } \\
\text { Normal IOP following iridectomy }\end{array}$ \\
$\begin{array}{l}\text { Elevated IOP following iridectomy with gonioscopic } \\
\text { pathology: }\end{array}$ & & \\
$\begin{array}{l}\text { Quarter to half of angle closed by goniosynechiae } \\
\text { More than half of angle closed by goniosynechiae }\end{array}$ & 14 & \\
$\begin{array}{l}\text { Visible damage to angle without goniosynechiae } \\
\text { Elevated IOP with normal angle: }\end{array}$ & 15 & -57 \\
$\begin{array}{l}\text { Misdiagnosed open-angle glaucoma } \\
\text { Possible mixed glaucoma }\end{array}$ & 3 & \\
\hline & 6 & -5 \\
\hline
\end{tabular}


of pigment covering all of the angle structures including Schwalbe's line, the anterior and posterior parts of the trabecular meshwork, the scleral spur, and the ciliary body. This distribution of pigment distinguishes a damaged angle from a heavily pigmented but otherwise normal angle in which pigment is precipitated on Schwalbe's line and the functional, posterior part of the trabeculum.

In 9 of the 267 operated eyes the IOP remained elevated after iridectomy in spite of an open, apparently normal angle. In 3 of these eyes the preoperative picture was that of elevated IOP with a narrow (grade 1) but open angle, and the final diagnosis was therefore open-angle glaucoma. In 6 eyes (of 3 patients) the preoperative IOP was between 23 and $32 \mathrm{mmHg}$, the anterior chamber was narrow grade 1 , there was no history of acute angle-closure, and one or more provocative test was strongly positive (elevation of IOP of 10 to $22 \mathrm{mmHg}$ during the test). In 2 eyes the mydriatic test was positive, in 2 eyes the prone-position test (Hyams et al., 1968) was positive, and in 2 eyes both the dark-room and prone-position tests were positive. In all of these 6 eyes the IOP not only remained elevated but was completely uninfluenced by the performance of an iridectomy. These 6 eyes should therefore be regarded as having possible mixed glaucoma, since the correct diagnosis could also be open-angle glaucoma with false-positive provocative tests.

\section{Discussion}

The prevalence of open-angle glaucoma in subjects over the age of 40 years is 0.5 to $0.6 \%$ (Pollack, 1968; Hyams et al., Keroub, 1976). One would therefore expect open-angle glaucoma to be a factor in not more than $0.6 \%$ of patients with angleclosure glaucoma.

Abrams (1961) found that 47 out of 1861 consecutive cases of primary glaucoma had been diagnosed as mixed glaucoma but that the diagnosis could be substantiated in only 5 patients-after careful reanalysis with particular attention to a history of haloes and attacks, the dark-room test, and (in 2 cases) the response to iridectomy.
The diagnosis of mixed glaucoma is complicated by the possibility that repeated episodes of angle closure may cause damage to the trabecular meshwork, which is not gonioscopically visible. Such damage would reduce the outflow facility and introduce an element of apparent open-angle glaucoma (Becker and Shaffer, 1965; Chandler and Grant, 1965).

In the present study we found visible signs of damage to the anterior chamber angle in 15 eyes in which no goniosynechiae were present. In the absence of visible evidence of angle damage these 15 eyes would have been diagnosed as having mixed glaucoma.

Out of the 267 eyes investigated 6 may have mixed glaucoma $(2 \cdot 2 \%)$. This figure must be regarded as a maximum, since in all 6 eyes the angle-closure element was diagnosed on the basis of provocative tests, which can occasionally produce false positive results. In none of the 6 eyes was there any other direct evidence of angle-closure.

The study confirms the concept that mixed glaucoma is not a separate entity but a chance coexistence of open-angle and angle-closure glaucoma. In the interests of clear thinking it is important to distinguish between the rare cases of mixed glaucoma and the commoner cases of primary glaucoma of uncertain classification. From the practical, therapeutic point of view such a distinction is less important, since the treatment of choice in both cases is peripheral iridectomy followed if necessary by the use of the full range of antiglaucoma drugs and, occasionally, by a filtering procedure.

\section{References}

Abrams, J. D. (1961). British Journal of Ophthalmology, 45, 503.

Becker, B., and Shaffer, R. N. (1965). Diagnosis and Therapy of Glaucoma, p. 216. St Louis, Mosby.

Chandler, P. A., and Grant, W. M. (1965). Lectures on Glaucoma, p. 193. Philadelphia, Lea \& Febiger.

Hyams, S. W., Friedman, Z., and Neumann, E. (1968). American Journal of Ophthalmology, 66, 661.

Hyams, S. W., et al. (1976). Public Health (Jerusalem), p. 89 (in Hebrew), May 1976.

Pollack, I. P. (1968). Survey of Ophthalmology, 13, 4.

Shaffer, R. N. (1960). Transactions of the American Ophthalmological Society, 58, 219. 